\title{
A study of the optical band gap of zinc phthalocyanine nanoparticles using UV-Vis spectroscopy and DFT function
}

\author{
Khalil J. Hamam ${ }^{1}$ Mohammed I. Alomari ${ }^{2}$
}

Received: 26 March 2017/Accepted: 21 April 2017/Published online: 27 April 2017

(c) The Author(s) 2017. This article is an open access publication

\begin{abstract}
In the present work, we used the ultravioletVisible (UV-Vis) spectroscopy technique to find the optical band gap of zinc phthalocyanine nanoparticles (ZnPcNP) experimentally. Moreover, we used a time-dependent density functional theory (TDDFT) to simulate the UV-Vis absorption spectrum of $\mathrm{ZnPc}$ molecule in gas and solution phases. The ZnPc-NP absorption spectrum shows a shift toward higher energies compared to the bulk $\mathrm{ZnPc}$. The simulated UV-Vis and the experimental nanoparticle's spectrum were found to have a good agreement. The $\mathrm{ZnPc}$ energy band gap from the DFT calculations shows how it's possible to get wider range of energy band gap for the $\mathrm{ZnPc}$. The ZnPc-NP's size and shape were examined using the transmission electron microscope (TEM).
\end{abstract}

Keywords Zinc phthalocyanine - Nanoparticles · Band gap energy $\cdot$ Density functional theory (DFT)

\section{Introduction}

Preparation and characterization of nanostructure systems during the past decades has got scholars' attention due to their interesting optical, electronic and chemical properties (Weller 1996; Alivisatos 1996). Scholars focused on studying the nanoparticles derived from metals and

Khalil J. Hamam

hamam73@yahoo.com; khalil@ttu.edu.jo

1 Applied Physics Department, Faculty of Science, Tafila Technical University, P.O. Box 179, Tafila 66110, Jordan

2 Department of Chemistry and Chemical Technology, Faculty of Science, Tafila Technical University, P.O. Box 179, Tafila 66110 , Jordan inorganic semiconductors, due to their significant electrical and optical properties compared to bulk materials (Murray et al. 1993; Braum et al. 1996; Micic et al.1995; Heath et al. 1994).

As the dimension of a material gets smaller, the physical and optical properties were observed to change; such as the absorption spectrum of the material (Chen et al. 1994). We can observe this effect obviously in exotic colors, which is reflected in their absorption and emission spectra; this effect was attributed to the gradual conversion of the material edges into sharp and discrete molecular (excitonic) absorption bands; therefore, band gap becomes more larger, which is also known as the size quantization effect (Smith and Nie 2010).

The control of material's band gap energy, is an important issue in the semiconductor properties and nanoparticles applications (Dabbousi et al.1995; Colvin et al.1994). The theoretical band gap can be represented by Eq. 1 (Yoffe 1993):

$E_{\mathrm{g}}^{\mathrm{NP}}=E_{\mathrm{g}}^{\mathrm{bulk}}+\frac{h^{2} \pi^{2}}{2 R^{2}}\left(\frac{1}{m_{\mathrm{e}}^{*}}+\frac{1}{m_{\mathrm{h}}^{*}}\right)-\frac{1.884 e^{2}}{4 \pi \varepsilon R}$

where $E_{\mathrm{g}}^{\text {bulk }}$ is the bulk band gap. $R, m_{\mathrm{e}}^{*}, m_{\mathrm{h}}^{*}$ and $\varepsilon$ are the radius of the nanoparticle, electron's effective mass, hole's effective mass, and the dielectric constant, respectively. The third term in Eq. 1 arises due to the columbic attraction which is usually ignored in a high dielectric constant medium. Equation 1 shows that as the material's size gets smaller, its band gap energy gets higher. This formula is used to estimate the band gap for an isotropic spherically nanostructures. Therefore, values obtained for the energy band gap from Eq. 1 are usually over estimated (Li et al. 2001).

Zinc phthalocyanine $(\mathrm{ZnPc})$ is an organic semiconductor compound (Eley 1948), which is used as a photosensitizer 
for Photodynamic therapy (PDT) (Fadel et al. 2010; Ping et al. 2016); and in optoelectronic applications such as organic light-emitting diodes (OLED) (Run-Da et al. 2013; Heo et al. 2013) and organic photovoltaics (OPVs) (Kim and Bard 2004). The size effect on the electrical and optical properties of metal-free phthalocyanine nanoparticles have been studied using the physical method such as mechanical grinding, it's found that as the material's size gets smaller their photoconductivity efficiency was improved (Saito et al. 1991). However, fabrication of very tiny homogeneous nanoparticles of MPc's by using the physical methods (grinding method) is not feasible. Therefore, other methods have been developed to fabricate a tiny size of organic nanoparticles such as liquid phase direct precipitation (LPDP) (Chen et al. 1991), laser ablation (Tamaki et al. 2002) and liquid-liquid interface recrystallization techniques (LLIRCT) [Patil et al. 2007].

The spectroscopic study of metal phthalocyanine compounds (MPc) gives very useful information about the charge transfer between the central metal and the phthalocyanine ligand, as a result of the overlapping between their energy wave functions. One of the easiest ways to determine the optical energy band gap of the material is using their absorption spectrum.

In the present work, we studied the zinc phthalocyanine bulk and nanoparticles thin film's energy band gap using a simple technique, which is the UV-Vis absorption spectrum technique. The objective of this work is to synthesize the zinc phthalocyanine nanoparticles (ZnPc-NP) to study the size effect on the absorption spectrum and calculate their band gap energy. Additionally, density functional theory (DFT) calculations were used to find the minimum energy and optimized geometry for $\mathrm{ZnPc}$ molecule, and to simulate the expected UV-Vis absorption spectrum. Nanoparticles's size and shape were examined using the transmission electron microscope (TEM) images.

\section{Experimental and theoretical work}

\section{Synthesis of zinc phthalocyanine nanoparticles (ZnPc-NP)}

Zinc phthalocyanine (ZnPc) (99\% purity) and cetyltrimethylammonium bromide (CTAB) were purchased from Sigma-Aldrich, through a local chemical provider. The surfactant was added to $(150 \mathrm{ml})$ distilled water and stirred at $50{ }^{\circ} \mathrm{C}$ until a transparent solution was observed. $\mathrm{ZnPc}(0.05 \mathrm{~g})$ was dissolved in $17 \mathrm{ml}$ concentrated sulfuric acid $(98 \%)$, then the solution was added dropwisely, until a dark blue colloidal solution was obtained into an aqueous surfactant solution in an ice-methanol-water bath to keep the temperature of the solution at $0{ }^{\circ} \mathrm{C}$, the solution was under vigorous magnetic stirring. The resulted solution was washed to neutral with water using an ultra-filter. A trace amount of sulfuric acid was removed by anion exchange resin (Wang et al. 1999). The precipitate of the MPc nanoparticles was washed with water and acetone to remove any residual surfactants. ZnPc nanoparticles were dispersed into Dimethylformamide (DMF) and then spin coated at low speed (500 rpm for $40 \mathrm{~s}$ ) over clean glass substrates, to fabricate thin films. The thin films were annealed at $200{ }^{\circ} \mathrm{C}$ to remove the solvent.

\section{Characterization}

The UV-Vis spectrum was measured using Shimadzu UVVis spectrometer. UV-Vis is an easy and straightforward technique to determine the band gap energy of organic $\pi$ conjugated systems. The optical band gap can be estimated according to Eq. 2 (Costa et al. 2016):

$E_{\mathrm{g}}=\frac{1242}{\lambda_{\mathrm{th}}}$

where $E_{\mathrm{g}}$ represents the optical energy band gap (in $\mathrm{eV}$ ) and $\lambda_{\text {th }}$ represents the threshold wavelength (in $\mathrm{nm}$ ), obtained from the onset of the absorption spectrum (Bhadwal et al. 2014). The transmission electron microscope (TEM) images were prepared on carbon grids and scanned using Hitachi H-7000, to examine the size and the shape of our nanoparticles.

\section{Theoretical calculations}

The DFT Calculations were carried out using Gaussian09 package (Frisch et al. 2010). The applied basis sets are 6-31G(d) and the pseudo-potentials LANL2DZ. The 6-31G(d) (H, C, and $\mathrm{N}$ atoms) sets has the polarization function, which it is suitable for atoms that fill $s$ and $p$ atomic orbitals. The pseudo-potentials LANL2DZ is used for heavy atoms such as $\mathrm{Zn}$ atom. The $\mathrm{ZnPc}$ structure has been optimized using the B3LYP function. To investigate the UV spectra we have employed time-dependent density functional theory TDDFT on the optimized structure in gas and DMF solvent. Moreover, we have reported the energy of the molecular orbitals and then calculated the $\Delta E$ (gap) between the frontier HOMO-LUMO orbitals and between molecular orbitals of main peaks of the spectra.

\section{Results and discussion}

Organic molecules of phthalocyanine and their derivatives show optical characteristics because of their ring structure. They have two types of energy bands; B-band $(\gamma$ or Soret band) and Q-band ( $\alpha$ band in porphyrin) (Stillman and 
Nyokong 1989). The zinc phthalocyanine bulk absorption spectrum and the zinc phthalocyanine nanoparticles thin films are shown in Fig. 1. For bulk material, the B-band shows the maximum peak at $332.5 \mathrm{~nm}$. The peak at $618 \mathrm{~nm}$ in the Q-band energy was assigned to the first $\pi-\pi^{*}$ transition on the phthalocyanine macrocycle (Davidson 1982) and the shoulder at $676 \mathrm{~nm}$ could be assigned to an exciton state (Mack and Stillman 1997). ZnPc-NP

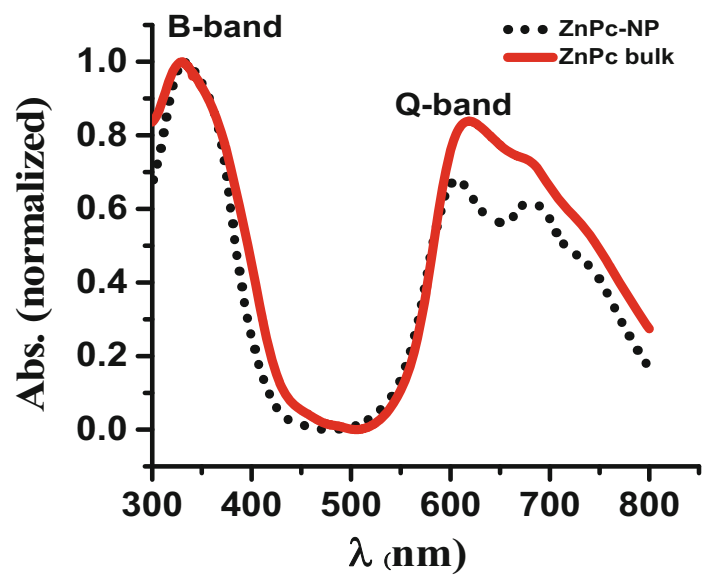

Fig. 1 The absorption spectrum for $\mathrm{ZnPc}$ nanoparticles $\mathrm{ZnPc}-\mathrm{NP}$ (short dot line) and bulk $\mathrm{ZnPc}$ (solid line) absorption spectrum shows a shift of the absorption peaks toward the short wavelength region (blue shift). In the B-band region, the $332.5 \mathrm{~nm}$ peak was shifted to the $330.5 \mathrm{~nm}(\Delta \lambda=2 \mathrm{~nm})$; while for the $618 \mathrm{~nm}$ peak had been shifted to $605.5 \mathrm{~nm}$ in the nanoparticles spectrum, which is considered as the largest shift in the wavelengths $(\Delta \lambda=12.5 \mathrm{~nm})$, among all of them. The blue shift of $\lambda$ can be described like "quantum size effect" in semiconductor nanoparticles (Laurs and Heiland 1987). The $676 \mathrm{~nm}$ shoulder was developed into a clear, broad peak (677-691 nm). ZnPc-NP absorption spectrum shows the broadening spectrum compared to the bulk ZnPc, which could be attributed to a change in the type of molecules stacking due to the formation of the nanoparticles (Masuhara et al. 2003).

The optical band gap was determined from the analysis of the absorption spectrum as described by Tauc plot using the formula (El-Nhass et al. 2001)

$\alpha h v=\alpha_{o}\left(h v-E_{\mathrm{g}}\right)^{n}$

where $h v$ is the energy of incident photons and $E_{\mathrm{g}}$ is the value of the optical band gap corresponding to transitions indicated by the value of $n . \alpha_{\mathrm{o}}$ is a constant which depends on the transition probability. A best linear fit was found for $n=0.5$ which indicates an allowed direct transitions in the material (Kim et al. 2012). The extrapolation of the Tauc
Fig. 2 Determination of the band gap of a bulk ZnPc and b ZnPc-NP using experimental data $\mathbf{c}$ gas phase and $\mathbf{d} \mathrm{DMF}$ solvent using DFT data
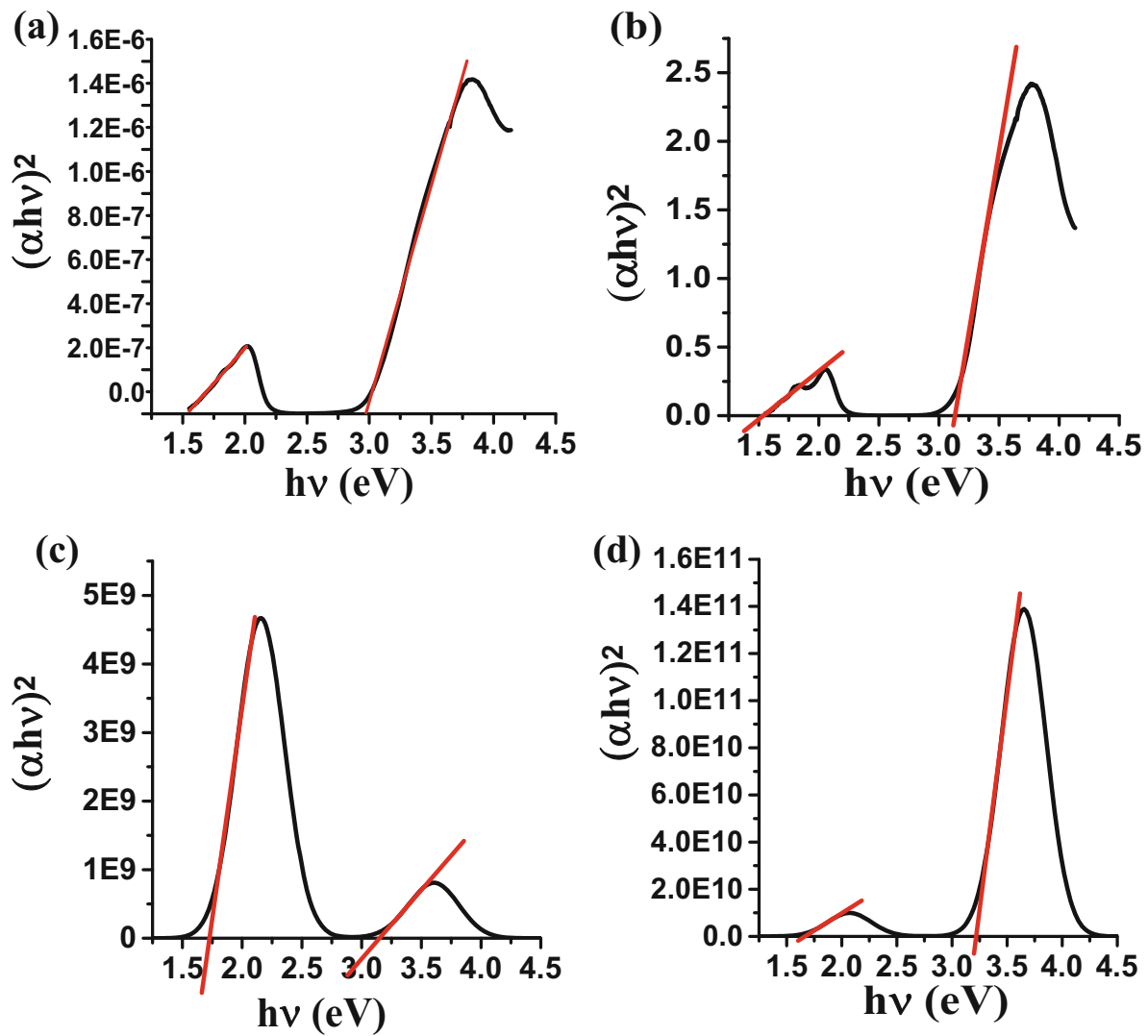
Table 1 Band gap and threshold wavelengths value for bulk $\mathrm{ZnPc}$, $\mathrm{ZnPc}-\mathrm{NP}$ and $\mathrm{ZnPc}-\mathrm{DFT}$

\begin{tabular}{llllll}
\hline Material & \multicolumn{2}{l}{ B-band } & & Q-band \\
\cline { 2 - 3 } & $E_{\mathrm{g}}(\mathrm{eV})$ & $\lambda_{\text {th }}(\mathrm{nm})$ & & $E_{\mathrm{g}}(\mathrm{eV})$ & $\lambda_{\text {th }}(\mathrm{nm})$ \\
\hline ZnPc bulk & 2.97 & 418 & & 1.53 & 812 \\
ZnPc-NP & 3.13 & 396 & & 1.55 & 801 \\
ZnPc (DFT-gas) & $3.15^{\mathrm{a}}$ & 395.5 & & $1.70^{\mathrm{a}}$ & 776.3 \\
ZnPc (DFT-DMF) & $3.20^{\mathrm{b}}$ & 394.3 & & $1.65^{\mathrm{b}}$ & 757.3 \\
\hline
\end{tabular}

a From Tauc plot Fig. 2c

b Fig. 2d

plot to the abscissa gives the value of the optical band gap. The values of the energy band gaps for the bulk material and the nanoparticles of $\mathrm{ZnPc}$ in the two regions are shown in Fig. 2a, b. The DFT data was used to figure out the band gap energy and threshold wavelength. The Tauc plot extracted from DFT calculations, in gas phase and in DMF solvent are shown in Fig. 2c, d. Band gap values that have been extracted from the experimental and the theoretical data at and threshold wavelengths are listed in Table 1.

The $\mathrm{ZnPc}$ nanoparticles average size and shape were determined by using transmission electron microscope (TEM). Almost an oval geometry (non-spherical shape) of the nanoparticles was observed. Furthermore, clustering and aggregation effect was observed in our samples as shown in Fig. 3, left. The average calculations of the nanoparticle's diameter were in the order of $6.5 \pm 0.3 \mathrm{~nm}$ as shown in Fig. 3, right

Density functional theory (DFT) study on $\mathrm{ZnPc}$ was performed, to find the estimated $\mathrm{ZnPc}$ band gap for a single molecule in gaseous and solution (DMF) phase, for comparison purposes with the experimental nanoparticle's band

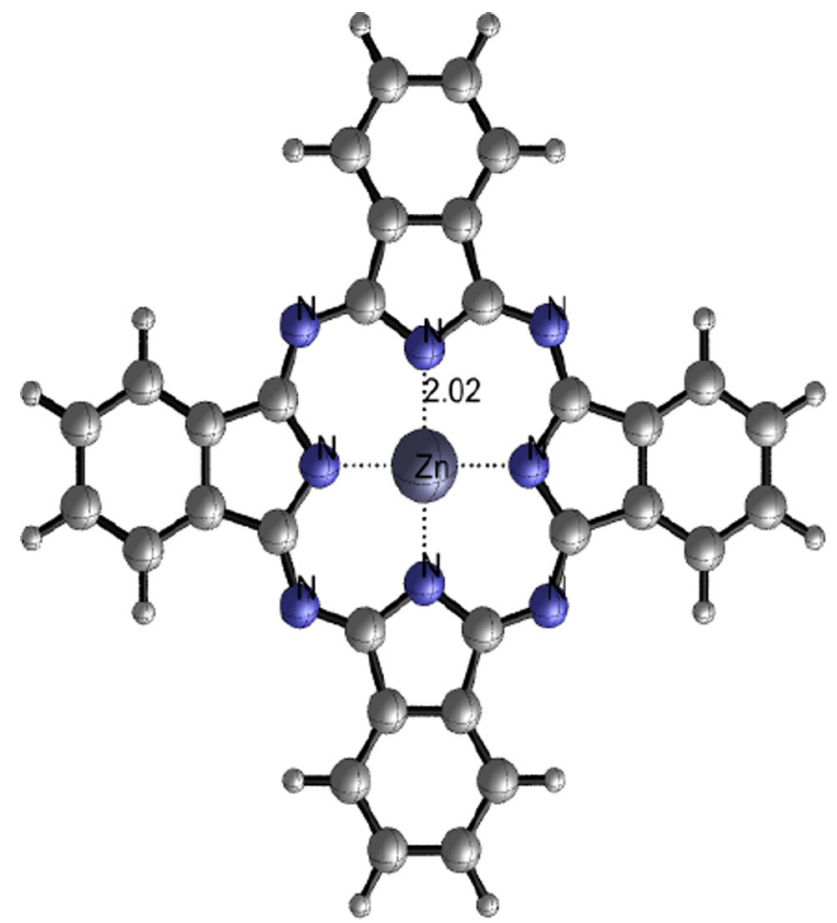

Fig. 4 The planar structure of the zinc phthalocyanine $\mathrm{ZnPc}$ molecule. The $\mathrm{Zn}$ and $\mathrm{N}$ atoms are labeled, $\mathrm{C}$ atoms are in gray. The $\mathrm{Zn}-\mathrm{N}$ bond length of the optimized structure is $2.02 \AA$. [The chemical formula is $\mathrm{C}_{32} \mathrm{H}_{18} \mathrm{~N}_{8} \mathrm{Zn}$ and the atomic weight is $579.942 \mathrm{~g} / \mathrm{mol}]$

gap. The optimized structure of $\mathrm{ZnPc}$ is shown in Fig. 4 which was a planar structure.

In the Q-band the electronic transition occurs from HOMO, which has an electronic density mainly located on the phthalocyanine molecule, to the LUMO, which has a small electronic density on $\mathrm{Zn}-\mathrm{N}$ bond as shown in Fig. 5 . The calculated energy gap is $E_{\mathrm{g}}=2.23 \mathrm{eV}$. The B-band electronic transition occurs between HOMO-4/LUMO
Fig. 3 TEM image of the $\mathrm{ZnPc}$ nanoparticles (left) and the histogram for particles frequency and the average diameter of the particles (right); the average diameter was $6.5 \pm 0.3 \mathrm{~nm}$
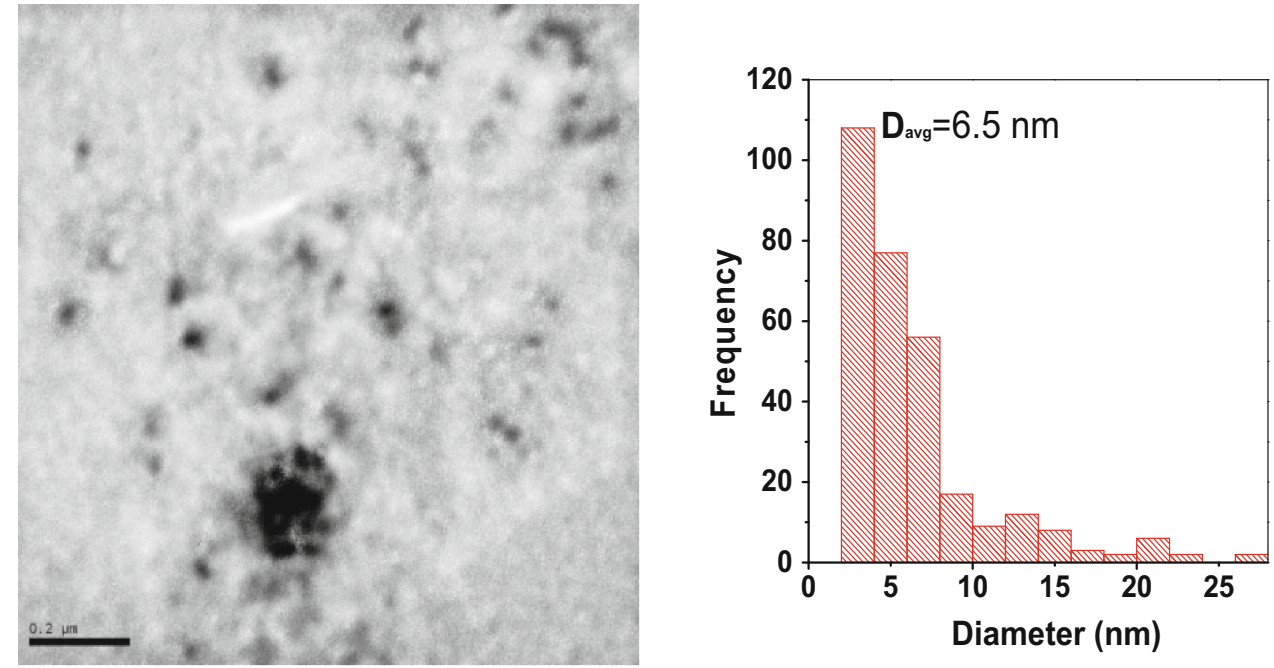

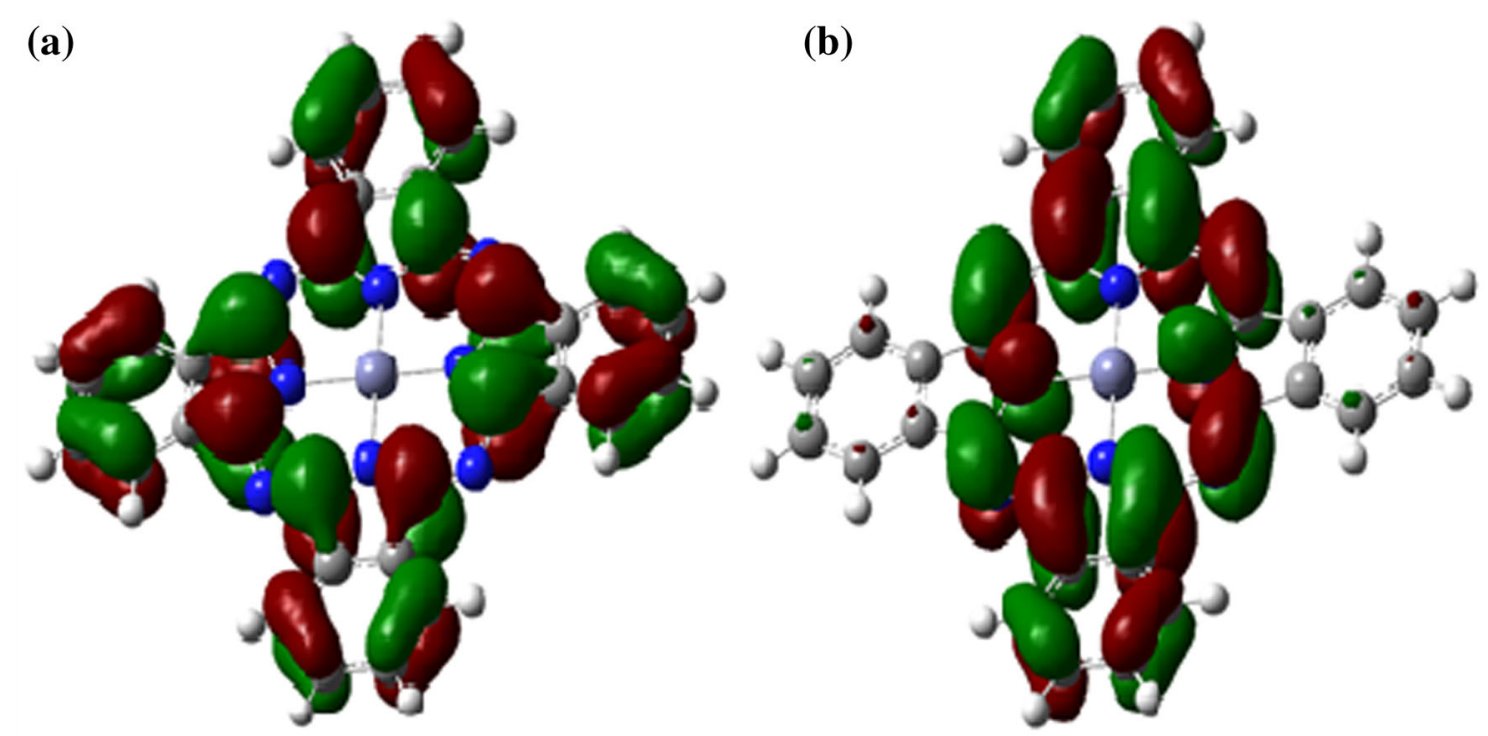

Fig. 5 Virtual a LUMO and b HOMO frontier orbitals of $\mathrm{ZnPc}$ molecule, the energy gap is $E_{\mathrm{g}}=2.23 \mathrm{eV}$

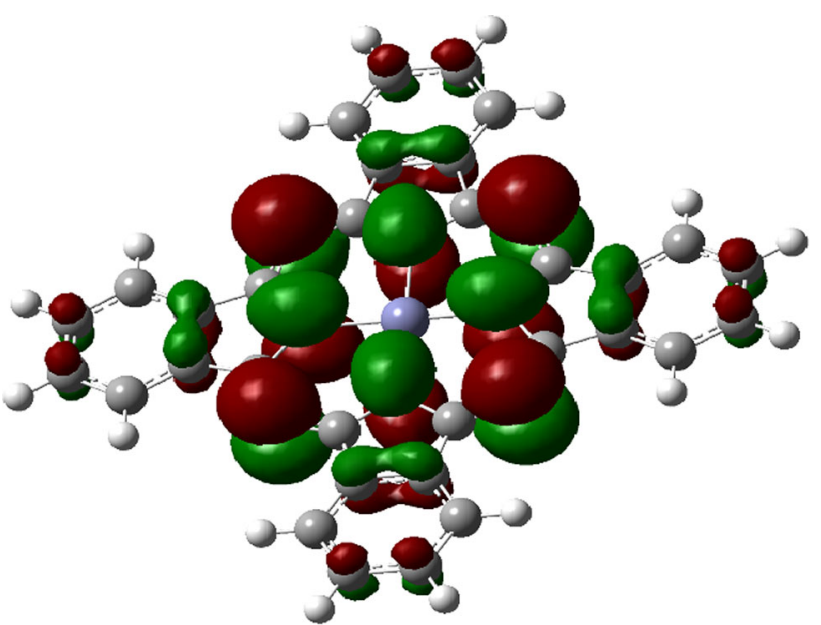

Fig. 6 The HOMO-4 orbital, where B-band electronic transition occurs, $E_{\mathrm{g}}=3.91 \mathrm{eV}$

orbitals, $E_{\mathrm{g}}=3.91 \mathrm{eV}$ as shown in Fig. 6. The electrostatic potential surface and contours of $\mathrm{ZnPc}$ is shown in Fig. 7, where the $\delta+$ and $\delta-$ charges are residing on $\mathrm{Zn}$ and on $\mathrm{N}$-atoms, respectively. The electrostatic potential surfaces can give us an idea about how the $\mathrm{ZnPc}$ molecules are stacking in the nanostructure system. The most probable aggregation in the system is the $\mathrm{H}$-aggregation due to high energy shift in the absorption spectrum and the information provided by the electrostatic potential distribution on the $\mathrm{ZnPc}$ molecule.

The UV-Vis simulation was performed after optimizing the molecule geometry at minimum energy (no imaginary
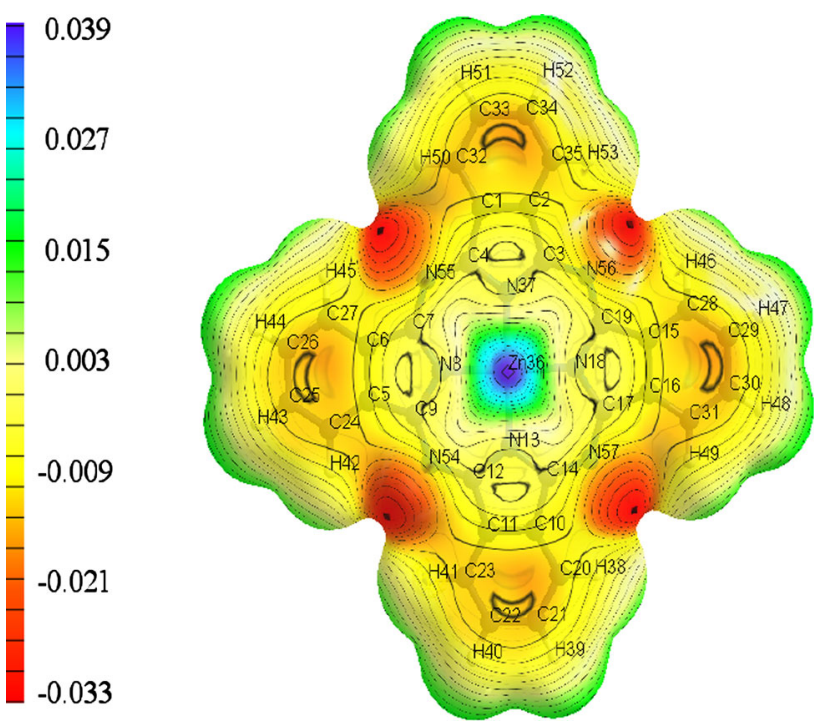

Fig. 7 The electrostatic potential surface and contours of ZnPc, the $\delta^{+}$charge on $\mathrm{Zn}$ and $\delta^{-}$charge on $\mathrm{N}$-atoms

frequency) or transition states (a single imaginary frequency) as shown in Fig. 8. The simulated UV-Vis shape has a good agreement with the experimental spectra, the B-band and Q-band were located in the same range of wavelength as we observed in the experimental spectra. The position of the maximum peaks is listed in Table 2 . Furthermore, the associated calculations of the density of states of the ZnPc are shown in Fig. 9 in gas phase (a) and of the DMF solvent (b) which shows the virtual and occupied orbitals. 


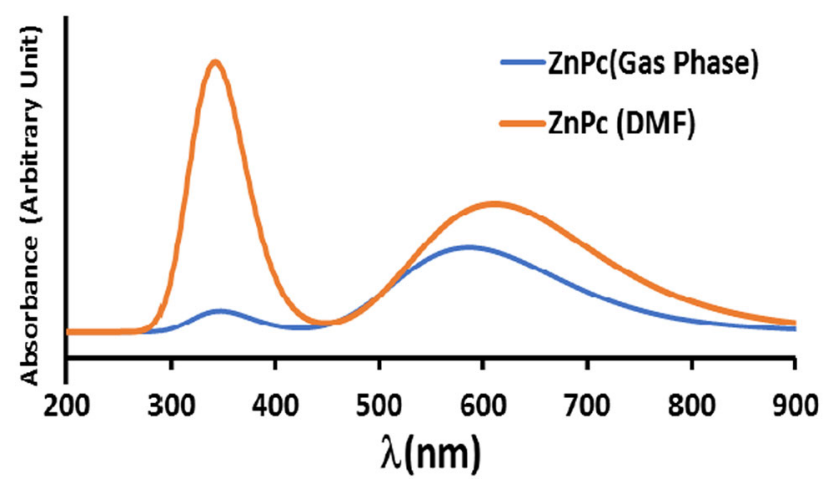

Fig. 8 The UV-Vis spectra of ZnPc molecule using TD-DFT theory, in the gas phase and the DMF solvent

Table 2 Maximum values of wavelengths at each absorption peak for $\mathrm{ZnPc}$ molecule using DFT-B3LYP

\begin{tabular}{lll}
\hline & $\lambda_{\max }(\mathrm{nm})$ gas phase & $\lambda_{\max }(\mathrm{nm})$ DMF solvent \\
\hline B-band & 343.9 & 341.7 \\
Q-band & 586.3 & 611.2 \\
\hline
\end{tabular}

\section{Conclusions}

The UV-Vis technique was used to determine the band gap energy for bulk $\mathrm{ZnPc}$ and $\mathrm{ZnPc}$ nanoparticles (ZnPc-NP) thin films. The shift in the absorption position was considered as an evident of the formation of the nanoparticles. Nanoparticle's band gap energy recorded higher values at both B-band and Q-band (3.13 and $1.55 \mathrm{eV}$, respectively) compared to the values for similar bands of bulk $\mathrm{ZnPc}$ (2.97 and $1.53 \mathrm{eV}$, respectively). Therefore, ZnPc-NP's absorption spectrum shows a shift toward the higher energy photons; the $332.5 \mathrm{~nm}$ peak was shifted to the $330.5 \mathrm{~nm}$ (B-band region); and the $618 \mathrm{~nm}$ peak had been shifted to $605.5 \mathrm{~nm}$ (Q-band region). DFT calculations using the DFT-B3LYP method was used to find the band gap of $\mathrm{ZnPc}$ molecule and compared with the experimental value; DFT calculations show that the maximum tuned band width could reach $2.23 \mathrm{eV}$. The simulated UV-Vis has a good agreement in shape and position of B-band and Q-band with the experimental measurements. Transmission electron microscope (TEM) was used to examine the $\mathrm{ZnPc}$ nanoparticles size, they have an average diameter of $6.5 \pm 0.3 \mathrm{~nm}$. Controlling the band gap of the organic
Fig. 9 The density of states and the band structure of $\mathrm{ZnPc}$ calculated, $\mathbf{a}$ in gas phase and b in DMF solvent
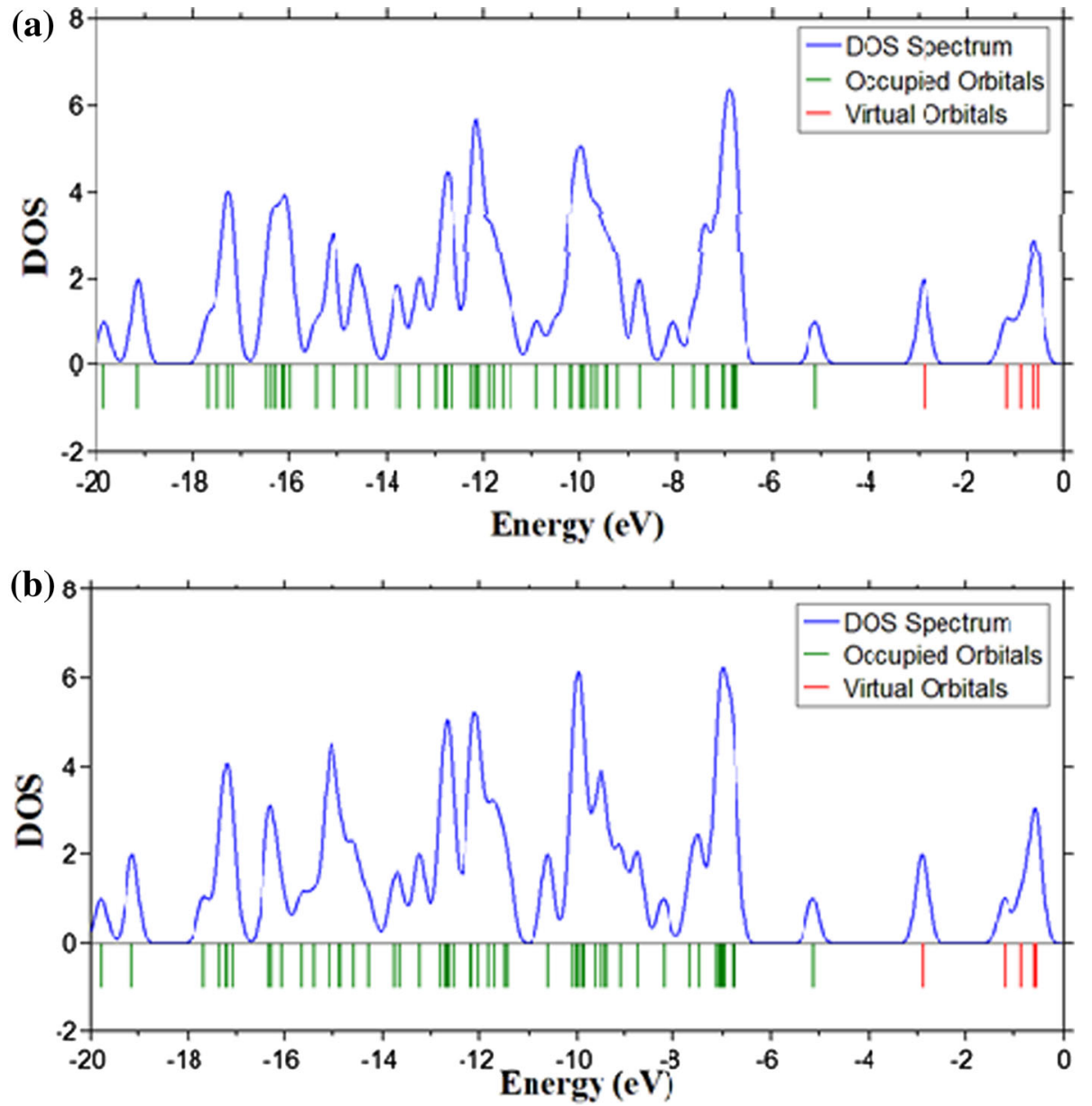
semiconductors is an important issue, for applications such as light emitting diode, organic photovoltaic devices and the photodynamic therapy as a photosensitizer.

Acknowledgements We would like to submit our gratitude to Dr. Anas Lataifeh, Dr. Salim Khalil and Dr. Ghassab Al-Mazaideh from chemistry department; for their assistant and the their valued comments.

Open Access This article is distributed under the terms of the Creative Commons Attribution 4.0 International License (http:// creativecommons.org/licenses/by/4.0/), which permits unrestricted use, distribution, and reproduction in any medium, provided you give appropriate credit to the original author(s) and the source, provide a link to the Creative Commons license, and indicate if changes were made.

\section{References}

Alivisatos AP (1996) Semiconductor clusters, nanocrystals, and quantum dots. Science 271:933-937. doi:10.1126/science.271. 5251.933

Bhadwal AS, Tripathi RM, Gupta RK, Kumar N, Singha RP, Shrivastav A (2014) Biogenic synthesis and photocatalytic activity of CdS nanoparticles. RSC Adv 4:9484-9490. doi:10. 1039/c3ra46221h

Braum PV, Osenar P, Stupp SI (1996) Semiconducting superlattices templated by molecular assemblies. Nature 380:325-328. doi:10. 1038/380325a0

Chen W, McLendon G, Marchetti A, Rehm JM, Michal IF, Myers C (1994) Size dependence of radiative rates in the indirect band gap material AgBr. J Am Chem Soc 116:1585-1586. doi:10. 1021/ja00083a060

Chen HZ, Pan C, Wang M (1991) Characterization and photoconductivity study of TiOPc nanoscale particles prepared by liquid phase direct reprecipitation. Nanostruct Mater 11:523-530. doi:10.1016/s0965-9773(99)00338-4

Colvin VC, Schlamp MC, Alivisatos AP (1994) Light-emitting diodes made from cadmium selenide nanocrystals and a semiconducting polymer. Nature 370:354-357. doi:10.1038/370354a0

Costa JCS, Taveira RJS, Lima CFRAC, Mendes A, Santos LMNBF (2016) Optical band gaps of organic semiconductor materials. Opti Mat 58:51-60. doi:10.1016/j.optmat.2016.03.041

Dabbousi BO, Bawendi MG, Onitsuka O, Rubner MF (1995) Electroluminescence from CdSe quantum-dot/polymer composites. Appl Phys Lett 66:1316. doi:10.1063/1.113227

Davidson AT (1982) The effect of the metal atom on the absorption spectra of phthalocyanine films. J Chem Phys 77:162. doi:10. 1063/1.443636

El-Nhass MM, Soliman HS, Metwally HS, Farid AM, Farag AAM, El Shazly AA (2001) Optical properties of evaporated iron phthalocyanine(FePc) thin films. J Opti 30:121-129. doi:10. 1007/bf03354732

Eley DD (1948) Phthalocyanines as semiconductors. Nature 162:819. doi:10.1038/162819a0

Fadel M, Kassab K, Fadeel DA (2010) Zinc phthalocyanine-loaded PLGA biodegradable nanoparticles for photodynamic therapy in tumor-bearing mice. Las Med Sci 25:283-292. doi:10.1007/ s10103-009-0740-x

Frisch MJ et al. (2010) Gaussian 09, Revision B.01, Gaussian, Inc., Wallingford CT
Heath JR, Shiang JJ, Alivisatos AP (1994) Germanium quantum dots: optical properties and synthesis. J Chem Phys 101:1067. doi:10. 1063/1.467781

Heo I, Kim J, Yim S (2013) Effect of incorporated PVP/Ag nanoparticles on $\mathrm{ZnPc} / \mathrm{C} 60$ organic solar cells. J Nanosci Nanotechnol 13:4316-4319. doi:10.1166/jnn.2013.7451

Kim JY, Bard AJ (2004) Organic donor/acceptor heterojunction photovoltaic devices based on zinc phthalocyanine and a liquid crystalline perylene diimide. Chem Phys Lett 383:11-15. doi:10. 1016/j.cplett.2003.10.132

Kim HJ, Kim JW, Lee HH, Lee B, Kim JJ (2012) Initial growth mode, nanostructure, and molecular stacking of a ZnPc:C60 bulk heterojunction. Adv Funct Mater 22:4244-4248. doi:10.1002/ adfm. 201200778

Laurs H, Heiland G (1987) Electrical and optical properties of phthalocyanine films. Thin Solid Films 149:129-142. doi:10. 1016/0040-6090(87)90288-4

Li L, Hu J, Yang W, Alivisatos AP (2001) Band gap variation of sizeand shape-controlled colloidal CdSe quantum rods. Nano Lett 1:349-351. doi:10.1021/n1015559r

Mack J, Stillman MJ (1997) Assignment of the optical spectra of metal phthalocyanine anions. Inorg Chem 36:413-425. doi:10. 1021/ic960737i

Masuhara H, Nakanishi H, Sasaki K (2003) Single organic nanoparticles. Springer-Verlag, Berlin. ISBN 978-3-642-62429-2

Micic OI, Sprague JR, Curtis CT, Jones KM, Machol JL, Nozik AJ, Giessen H, Fluegel B, Mohs G, Peyghambarian N (1995) Synthesis and characterization of InP, GaP, and GaInP2 quantum dots. J Phys Chem 99:7754-7759. doi:10.1021/j100019a063

Murray CB, Norris DJ, Bawendi MG (1993) Synthesis and characterization of nearly monodisperse $\mathrm{CdE}(\mathrm{E}=$ sulfur, selenium, tellurium) semiconductor nanocrystallites. J Am Chem Soc 115:8706-8715. doi:10.1021/ja00072a025

Patil KR, Sathaye SD, Hawaldar R, Sathe BR, Mandale AB, Mitra A (2007) Copper phthalocyanine films deposited by liquid-liquid interface recrystallization technique (LLIRCT). J Colloid Interface Sci 315:747-752. doi:10.1016/j.jcis.2007.07.031

Ping J, Peng H, Duan W, You F, Song M, Wang Y (2016) Synthesis and optimization of $\mathrm{ZnPc}$-loaded biocompatible nanoparticles for efficient photodynamic therapy. J Mater Chem B 4:4482-4489. doi:10.1039/c6tb00307a

Run-Da G, Shou-Zhen Y, Peng W, Yi Z, Shi-Yong L, Yu C (2013) Increased performance of an organic light-emitting diode by employing a zinc phthalocyanine based composite hole transport layer. Chin Phys B 22:127304. doi:10.1088/1674-1056/22/12/ 127304

Saito T, Kawanishi T, Kakuta A (1991) Photocarrier generation process of phthalocyanine particles dispersed in a polymer: effects of pigment particle size, polymer matrix and addition of fine $\gamma$-alumina particles. Jpn J Appl Phys A 30:1182. doi:10. 1143/jjap.30.11182

Smith AM, Nie N (2010) Semiconductor nanocrystals: structure, properties, and band gap engineering. Acc Chem Res 43:190-200. doi:10.1021/ar9001069

Stillman MJ, Nyokong T (1989) Phthalocyanines, properties and applications, ed. by C.C. Leznoff and ABP. Lever; Chapt. 3, VCD, New York, ISBN 1-56081-916-2

Tamaki Y, Asahi T, Masuhara H (2002) Nanoparticle formation of vanadyl phthalocyanine by laser ablation of its crystalline powder in a poor solvent. J Phys Chem A 106:2135-2139. doi:10.1021/jp012518a

Wang Y, Deng K, Gui L, Tang Y, Zhou J, Cai L, Qiu J, Ren D, Wang Y (1999) Preparation and characterization of nanoscopic organic semiconductor of oxovanadium phthalocyanine. J Coll Interface Sci 213:270-272. doi:10.1006/jcis.1999.6132 
Weller H (1996) Self-organized superlattices of nanoparticles. Angew Chem Int Ed Engl 35:1079-1081. doi:10.1002/anie.199610791

Yoffe AD (1993) Low-dimensional systems: quantum size effects and electronic properties of semiconductor microcrystallites (zero- dimensional systems) and some quasi-two-dimensional systems. Adv Phys 42(173):266. doi:10.1080/00018739300101484 